\section{Much ado about some thing}

The Nothing That Is: A Natural

\section{History of Zero}

by Robert Kaplan

Allen Lane: 1999. 246 pp. $£ 12.99$

\section{Ivor Grattan-Guinness}

This popular book deals with a fascinating aspect of mathematics: the occurrence of zero within the arithmetics of integers and real numbers, and its many representations as numerals. Robert Kaplan is at his best on the latter: a wide range of examples is given, both of numerals and also of possible origins for the signs ' 0 ' and ' 0 '. On this last point he gives more credit to the Greeks than do other authors, but as the history is so obscure no definitive position can be maintained. Kaplan is careful to stress that Indian mathematicians were the first to recognize that zero could act as a pukka number, and not only as a space filler to distinguish, say, 34 from 304 and 3,004, a practice that is at least Babylonian.

The author also covers related questions, such as possible values, if any, for $0 / 0$. He discusses the role of zero elsewhere in mathematics, for example with infinitesimals. Probability theory is omitted, however, although there are interesting questions about non-contradictory statements that nevertheless have value zero in a probability calculus.

On number systems, the main point of contrast is with roman numerals, where Kaplan rather exaggerates the difficulties of calculating in that system. He could also have made this contrast for us: that if today is Friday, then two days ago it was Wednesday, because we count today as the zeroth day; the the Roman equivalent was in calling Wednesday three days earlier.

The author notes the concern with 'nothing' in some of William Shakespeare's plays. The general implementation of the Hindu-Arabic numeral system occurred in Britain around Shakespeare's lifetime, and the playwright would not have missed the resemblance of ' 0 ' to the 'Wooden Oh' of his theatre building. Among modern examples of the cultural history of zero not noted here is John Cage's notorious musical composition Four Minutes, Thirty-Three Seconds, in which any number of musicians play nothing for this length of time. In lasting 273 seconds, the title surely alludes to $-273^{\circ}$ Celsius, the absolute zero of temperature. Another apparent candidate is Samuel Beckett's play Waiting for Godot, that is, God-O $=$ God-zero $=$ no God, one of the play's main themes.

The book is likely to be widely read; but its success is somewhat limited by several

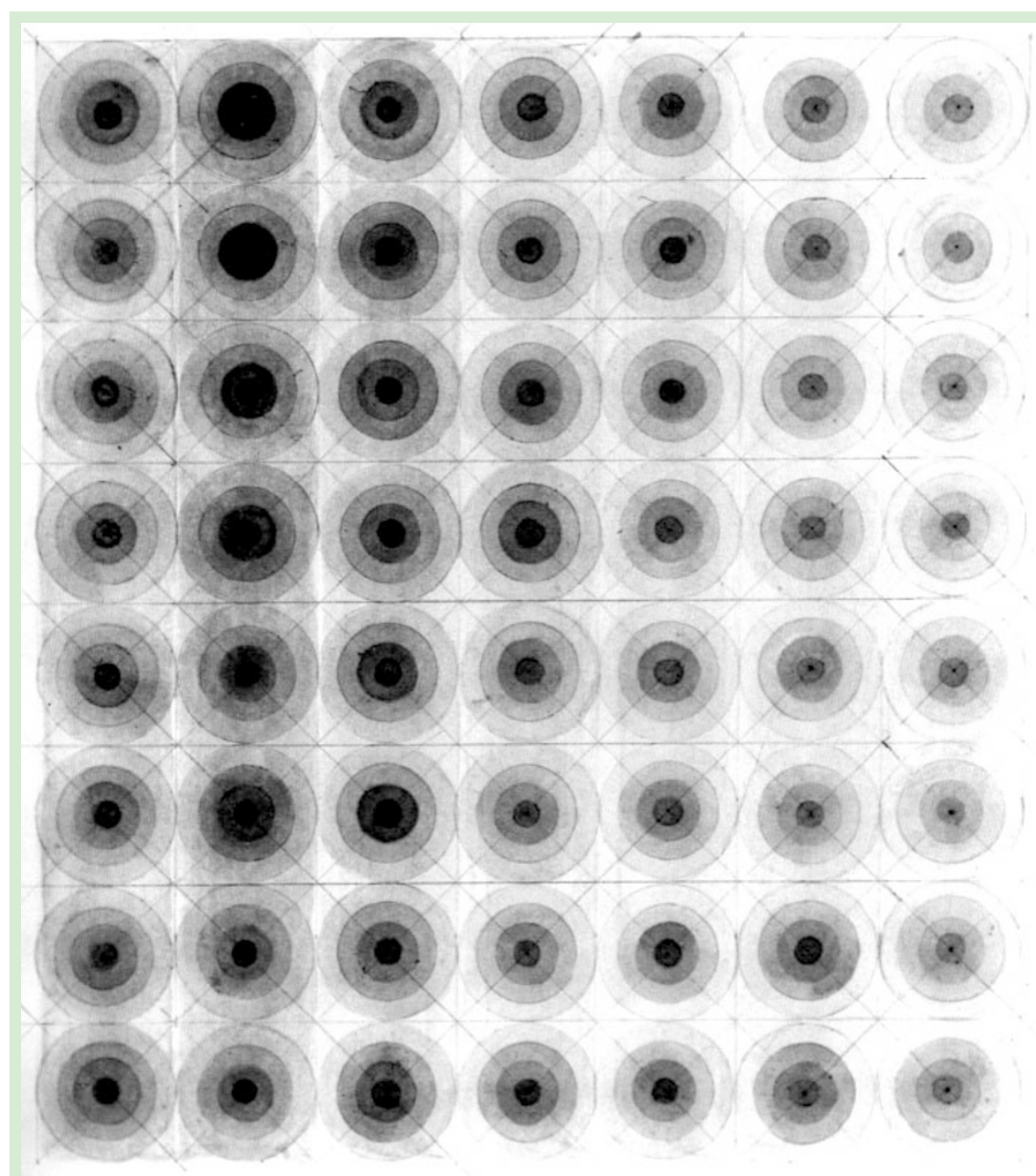

\title{
Zeroing in on process
}

Working in the late 1960s and early 1970s, a group of American artists rejected formal procedures in art. The result - process art was possibly the most ephemeral movement in art history; which seems appropriate for art that was centred more on the act than on the final product. The untitled work above, by Eva Hesse, is featured in the exhibition "Afterimage: drawing through process" (catalogue published in association with MIT Press, \$35, £24.50). The exhibition, which was organized by Cornelia Butler, has recently ended at the Museum of Contemporary Art in Los Angeles. It will be at the Contemporary Arts Museum, Houston, Texas, next year. considerations. Surely, the author missed a rare opportunity to explain the mistiming of the obsession with the end of the millennium. It does not happen this December, because when the Christian calendar system was instituted in the sixth century, no year zero was imposed; it is, I believe, unique as an application of the positive and negative integers in which zero is missing. The reason seems to have been misunderstanding zero to be nothing, a cultural distrust that was then related to views such as nature abhorring a vacuum. This is a fine example with which to emphasize that zero is some thing of a kind (for example, $7+0=7$, whereas $7+$ nothing is not defined), but the author makes little of it. In general, the difference between zero and nothing is not sufficiently emphasized.

Another of the book's limitations is in its treatment of negative numbers, which could have been given more attention. Like zero, negative numbers have been treated with mystification and distrust over the centuries, even though they have natural interpretations, such as directed distances ( +5 feet forwards, -5 feet backwards) and economic debt and credit (arguably, the origin of the sign '-' in the Late Middle Ages). Issues over the legitimacy of zero often were associated.

Kaplan frequently deploys nice analogies instead of explaining relevant features. For example, when describing the creation of the calculi by Isaac Newton and G. W. Leibniz, he correctly stresses the differences but does not explain the source of them, 
which lies in changing dimension. If one takes an infinite sequence of decreasing lines, then in the limit, one arrives at a point, and so changes from dimension 1 to dimension 0 . Newton followed this practice in defining his 'fluxion', although unclearly. By contrast, in Leibniz's theory, one adds to a given variable line $x$ an infinitesimally short 'differential' incremental line $\mathrm{d} x$, with $\mathrm{dd} x$ as its own increment, and so on; limits are explicitly avoided, and ' $\mathrm{d} y / \mathrm{d} x$ ' is the ratio, usually finite, of two differentials. This role of dimension could have been explained; instead, we learn about "the free play of mind, which browses on the pastures of phenomena", and find quotations from a novel and from Meister Eckhart.

More should have been said about set theory. For example, in the summary of nonstandard analysis as the modern theory of infinitesimals, there is a poem by Christian Morgenstern instead of a relevant explanation. Set theory has an empty set $\varnothing$, which is also not 'nothing': the author notes the definition of zero as the unit set of $\varnothing$, but he does not discuss the related tri-distinction between $\varnothing$, zero and nothing, which Bertrand Russell effected around 1900 (after anticipation by Gottlob Frege).

A similar non-nothing worth noting, and omitted in Kaplan's book, is the empty space, such as that between words. Their absence or presence is essential in contexts such as 'READINGANCIENTPAPYRI' and usingmodern.computerprogrammes. Also, the bibliography is available only on the World-Wide Web. This is a regrettable feature, for the book as it stands is useless, since no details can be checked or pursued in it.

Thus, The Nothing That Is is somewhat frustrating. The book as a whole is less than the sum of its parts - to invoke another fascinating mathematical topic with a long history, which has a zero and even a $0 / 0$ of its own with algebraic logicians such as George Boole and C. S. Peirce.

Ivor Grattan-Guinness in the Middlesex University Business School, Enfield, Middlesex EN3 4SF, UK.

\section{More mathematical somethings Discovering the Truth and Beauty of the Cosmos: A Mathematical Mystery Tour \\ by A. K. Dewdney \\ Wiley, £17.99, \$22.95}

\section{The Mathematics of Measurement: A Critical History}

by John J. Roche

Athlone, £55

\author{
Mathematical Sorcery: Revealing the \\ Secrets of Numbers \\ by Calcin C. Clawson \\ Perseus, $\$ 26.95$
}

\section{Science in culture}

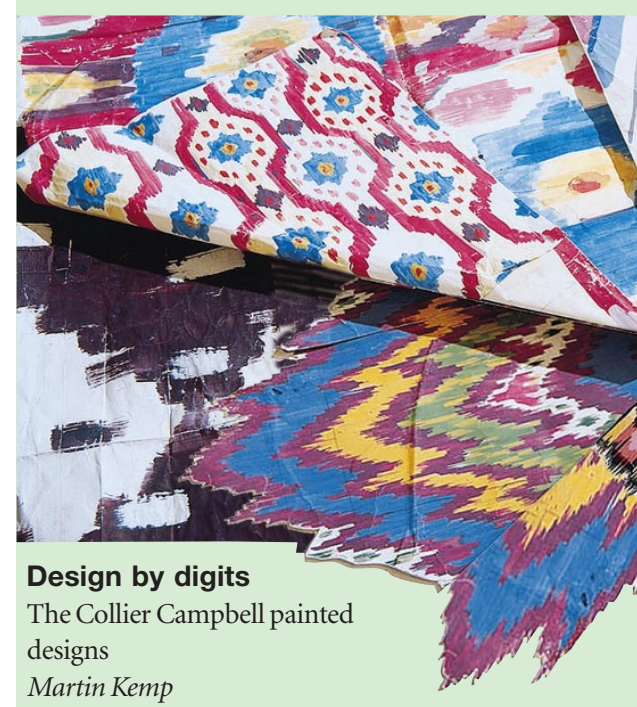

The origins of the term 'digital', which has come to signify the ultimate in depersonalized processing, lie in that most specifically human of instruments, the hand. The original 'digits' of computation were those ten fingers on which rudimentary acts of calculation were first performed. Now, in the age of computers, practitioners in almost every area of visual representation in art and science have witnessed severe polarization between designs arising from hand-eye coordination and the visual products of technology. The life's work of the remarkable design partnership, Collier Campbell (the archive of which is soon to be sold), proves that the divorce between the handmade and the machine-produced is not irrevocable.

Entering the world of textiles in the 1960s from engagement in painting rather than training in design techniques, the sisters Susan Collier and Sarah Campbell had a mission. It was to replace the tediously repetitive, graphically lifeless and coloristically crude textile patterns then generally available on the mass market with prints that retained the inherent life of freehand paintings. The motive was at once aesthetic and social, since they aspired to bring the costly and élite qualities of the individually made item within the reach of the widest possible public.

The painted designs that poured forth in a torrent of creative enthusiasm, in teemingly various styles, possess a vigour of mark-making, vitality of line and energy of colour more akin to a Matisse painting than to a stock 'repeat'. Indeed, they set out to "cheat the repeat", that is to say, to arrive at designs which, if repeated above and below, and on either side of one another, would flow dynamically across the whole surface, without the monotony of insistent repetitiveness.

Beginning commercial production with Liberty of London Prints in 1961, Collier (joined in 1968 by her sister) pushed printed textiles into new technical and visual realms. They insisted that printers exploit and extend their technologies to translate the painterly freshness

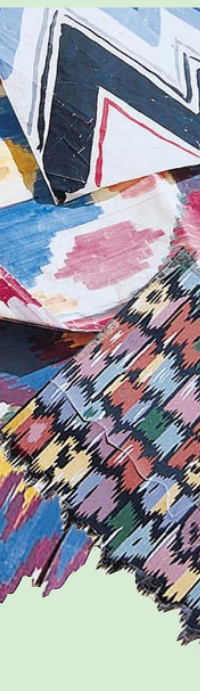
$\sqrt{1}$ s.

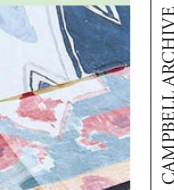

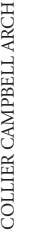
fabrics, so that they could
be reproduced without degradation of effect. The
premise on which they worked was that the eye can unerringly identify something that expressed the reach of hand, and that our aesthetic response to a hand-made item has its own unique character, something at once visual and somatic.

They were relying, without theory, on how the eye can undertake awesomely subtle acts of minute perceptual discrimination. These are the acts that allow us to recognize thousands of individual faces even from a fleeting glimpse of a mobile expression; to identify instantly a friend's handwriting on an envelope; and to distinguish a Leonardo da Vinci drawing from a pupil's pious copy. The tiny shadings of difference involved in such human discriminations are beyond description, relying upon what Collier Campbell term "inkling without words".

After almost 40 years of undiminished artistic fertility, working with leading international companies and fashion designers, Collier Campbell find themselves in a changed world. The present danger is, as they say, that "the hand can't be seen; only the price". The problem lies with the increasing domination of manufacturing and retail by a few conglomerates. The tendency has been to concentrate on the 'real estate' value of a few 'designer names' which are used to endorse a huge range of products, from perfumes to pants.

The core of the matter is not therefore a necessary incompatibility between hand and technology, between digits and digital, but how we can establish modes of practice that permit the visual traces of human presence to speak eloquently through the new technologies. The final product does need to be literally handmade, but if all we can offer in the popular market-place are mechanical designs devoid of human vibrancy, we will be impoverishing our visual culture. Martin Kemp is in the Department of the History of Art, University of Oxford, 35 Beaumont Street, Oxford OX1 2PG, UK. 